\title{
Infinite Sequence Solutions for Space-Time Fractional Symmetric Regularized Long Wave Equation
}

\author{
KANG Zhouzheng* \\ College of Mathematics, Inner Mongolia University for Nationalities, Tongliao 028043, \\ China.
}

Received 7 November 2015; Accepted 28 February 2016

\begin{abstract}
In this paper, we investigate the space-time fractional symmetric regularized long wave equation. By using the Bäcklund transformations and nonlinear superposition formulas of solutions to Riccati equation, we present infinite sequence solutions for space-time fractional symmetric regularized long wave equation. This method can be extended to solve other nonlinear fractional partial differential equations.
\end{abstract}

AMS Subject Classifications: 35A24, 35R11, 35-04

Chinese Library Classifications: O175.29

Key Words: Space-time fractional symmetric regularized long wave equation; Bäcklund transformations; nonlinear superposition formulas; exact solutions.

\section{Introduction}

In the past decades, much effort has been devoted to study nonlinear partial differential equations [1-25]. Nonlinear fractional partial differential equations (NFPDEs) regarded as the generalization form of nonlinear partial differential equations of integer order have attracted considerable attention in recent years. Moreover, the investigation of exact and approximate solutions for NFPDEs arising in mathematical physics, chemistry, biology, engineering, control theory, signal processing and so forth has become one of the most active and important research areas. A variety of analytical and numerical techniques have been well established and applied to solve NFPDEs, including the homogeneous balance method [6], the fractional sub-equation method [7-11], the exp-function method [12], the $\left(G^{\prime} / G\right)$-expansion method [13, 14], the first integral method [15], the modified trial equation method [16], the Jacobi elliptic equation method [17], the modified

${ }^{*}$ Corresponding author. Email address: zhzhkang@126.com (Z. Z. Kang) 
Kudryashov method [18], the homotopy analysis transform method [19], the fractional variational iteration method [20], the Adomian decomposition method [21], and so on. In many analytical methods, the fractional complex transformation proposed by $\mathrm{Li}$ and $\mathrm{He}$ [22] plays a key role in converting NFPDEs into NODEs. The purpose of present article is to examine the space-time fractional symmetric regularized long wave (FSRLW) equation by means of Riccati equation method and symbolic computation. As a result, based on the Bäcklund transformations and nonlinear superposition formulas of solutions to Riccati equation, infinite sequence solutions in terms of trigonometric and hyperbolic functions are established.

For the convenience of a reader, we recall the Jumarie's modified Riemann-Liouville derivative [23] of order $\alpha$, that is

$$
D_{t}^{\alpha} f(t)= \begin{cases}\frac{1}{\Gamma(-\alpha)} \int_{0}^{t}(t-\xi)^{-\alpha-1}(f(\xi)-f(0)) \mathrm{d} \xi, & \alpha<0, \\ \frac{1}{\Gamma(1-\alpha)} \frac{\mathrm{d}}{\mathrm{d} t} \int_{0}^{t}(t-\xi)^{-\alpha}(f(\xi)-f(0)) \mathrm{d} \xi, & 0<\alpha<1, \\ \left(f^{(n)}(t)\right)^{(\alpha-n)}, \quad n \leq \alpha<n+1, n \geq 1 . & \end{cases}
$$

Some significant properties of fractional modified Riemann-Liouville derivative are

$$
\begin{aligned}
& D_{t}^{\alpha} t^{\delta}=\frac{\Gamma(1+\delta)}{\Gamma(1+\delta-\alpha)} t^{\delta-\alpha}, \quad \delta>0, \\
& D_{t}^{\alpha}(f(t) g(t))=g(t) D_{t}^{\alpha} f(t)+f(t) D_{t}^{\alpha} g(t), \\
& D_{t}^{\alpha} f[g(t)]=f_{g}^{\prime}[g(t)] D_{t}^{\alpha} g(t)=D_{g}^{\alpha}[g(t)]\left(g^{\prime}(t)\right)^{\alpha} .
\end{aligned}
$$

The layout of this paper is as follows. In Section 2 and Section 3, we present the main steps of Riccati equation method, and list the Bäcklund transformations and nonlinear superposition formulas [24, 25] of solutions to Riccati equation. In Section 4, we apply this method to establish infinite sequence solutions for space-time FSRLW equation. The last section is the conclusion.

\section{Method}

Consider a NFPDE in three independent variables as

$$
P\left(u, D_{t}^{\alpha} u, D_{x}^{\beta} u, D_{y}^{\gamma} u, D_{t}^{\alpha} D_{t}^{\alpha} u, D_{t}^{\alpha} D_{x}^{\beta} u, \cdots\right)=0, \quad 0<\alpha, \beta, \gamma \leq 1,
$$

where $D_{t}^{\alpha} u, D_{x}^{\beta} u, D_{y}^{\gamma} u, \cdots$ are the modified Riemann-Liouville derivatives, and $P$ is a polynomial in $u$ and its fractional derivatives. We find solutions to Eq. (2.1) in the form

$$
u(t, x, y)=U(\xi), \xi=\frac{c t^{\alpha}}{\Gamma(1+\alpha)}+\frac{k x^{\beta}}{\Gamma(1+\beta)}+\frac{l y^{\gamma}}{\Gamma(1+\gamma)} .
$$


Then, Eq. (2.1) is reduced to a nonlinear ordinary differential equation

$$
O\left(U, U^{\prime}, U^{\prime \prime}, U^{\prime \prime \prime}, \cdots\right)=0,
$$

where the prime represents the derivative with respect to $\xi$. Next, we aim to find exact solutions for Eq. (2.2). We suppose that formal solution of (2.2) can be expressed by

$$
U(\xi)=A_{0}+\sum_{i=1}^{m}\left[w^{i-1}\left(A_{i} w+C_{i} \sqrt{R+w^{2}}\right)+B_{i} w^{-i}\right]
$$

in which $A_{0}, A_{i}, B_{i}, C_{i}(i=1,2, \cdots, m)$, and $R$ are constants to be determined. And $w=w(\xi)$ satisfies Riccati equation

$$
\frac{\mathrm{d} w}{\mathrm{~d} \xi}=R+w^{2}
$$

which possesses the following solutions.

For $R<0$, the expressions of hyperbolic function solutions read

$$
\begin{aligned}
& w_{0}(\xi)=-\sqrt{-R} \tanh (\sqrt{-R} \xi), \\
& w_{0}(\xi)=-\sqrt{-R} \operatorname{coth}(\sqrt{-R} \xi), \\
& w_{1}(\xi)=\frac{b_{3} R+a_{3} \sqrt{-R} \tanh (\sqrt{-R} \xi)}{-a_{3}+b_{3} \sqrt{-R} \tanh (\sqrt{-R} \xi)} .
\end{aligned}
$$

For $R>0$, the expressions of trigonometric function solutions read

$$
\begin{aligned}
& w_{0}(\xi)=\sqrt{R} \tan (\sqrt{R} \xi) \\
& w_{0}(\xi)=-\sqrt{R} \cot (\sqrt{R} \xi) \\
& w_{1}(\xi)=\frac{\sqrt{R}[\cos (\sqrt{R} \xi)+\sin (\sqrt{R} \xi)]}{\cos (\sqrt{R} \xi)-\sin (\sqrt{R} \xi)} \\
& w_{1}(\xi)=\frac{-(r \sqrt{R}+C R) \cos (\sqrt{R} \xi)+\sqrt{R}(r-C \sqrt{R}) \sin (\sqrt{R} \xi)}{(r-C \sqrt{R}) \cos (\sqrt{R} \xi)+(r+C \sqrt{R}) \sin (\sqrt{R} \xi)} \\
& w_{1}(\xi)=\frac{-3 b_{4} R+4 a_{4} \sqrt{R}-5 b_{4} R \sin (2 \sqrt{R} \xi)-5 a_{4} \sqrt{R} \cos (2 \sqrt{R} \xi)}{3 a_{4}+4 b_{4} \sqrt{R}+5 a_{4} \sin (2 \sqrt{R} \xi)-5 b_{4} \sqrt{R} \cos (2 \sqrt{R} \xi)} \\
& w_{1}(\xi)=\frac{-b_{5} R+a_{5} \sqrt{R}[\sec (2 \sqrt{R} \xi)+\tan (2 \sqrt{R} \xi)]}{a_{5}+b_{5} \sqrt{R}[\sec (2 \sqrt{R} \xi)+\tan (2 \sqrt{R} \xi)]} \\
& w_{1}(\xi)=\frac{\sqrt{R}\left[-2 a_{6} b_{6} \sqrt{R}+\left(a_{6}^{2}-b_{6}^{2} R\right)(\sec (2 \sqrt{R} \xi)+\tan (2 \sqrt{R} \xi))\right]}{a_{6}^{2}-b_{6}^{2} R+2 a_{6} b_{6} \sqrt{R}[\sec (2 \sqrt{R} \xi)+\tan (2 \sqrt{R} \xi)]}
\end{aligned}
$$

where $r, a_{i}, b_{i}(i=3,4,5,6)$ and $C$ are arbitrary nonzero constants. 
For $R=0$, the rational solution is

$$
w_{0}(\xi)=\frac{1}{-\xi+d_{0}}
$$

where $d_{0}$ is free constant.

Step 1. By homogeneous balance between the highest order derivative and nonlinear terms appearing in Eq. (2.2), one can get the value of $m$ easily.

Step 2. Substituting (2.3) together with (2.4) into Eq. (2.2), collecting all terms with the same powers of $w^{j_{1}}(\xi)$ and $w^{j_{2}}(\xi)\left(\sqrt{R+w^{2}(\xi)}\right)^{j_{3}}$, and equating zero of all the coefficients yield a system of algebraic equations about unknowns $\left\{A_{0}, A_{i}, B_{i}, C_{i}, R\right\}(i=1,2, \cdots, m)$.

Step 3. Solving the system obtained in Step 2 by symbolic computation system, one can get all the values of unknowns $\left\{A_{0}, A_{i}, B_{i}, C_{i}, R\right\}(i=1,2, \cdots, m)$. Inserting all the values of unknowns and solutions (2.5)-(2.15) into (2.3), many families of exact solutions to (2.1) can be got.

\section{Bäcklund transformations and nonlinear superposition formulas of solutions to Eq. (2.4)}

\subsection{Bäcklund transformations}

Eq. (2.4) admits Bäcklund transformations

$$
\tilde{w}(\xi)=\frac{p_{2}+q_{2} w(\xi)+m_{2} w^{2}(\xi)+r_{2} w^{\prime}(\xi)+n_{2} w^{3}(\xi)+l_{2}\left(w^{\prime}(\xi)\right)^{2}}{a_{2}+b_{2} w(\xi)+d_{2} w^{2}(\xi)+c_{2} w^{\prime}(\xi)+f_{2} w^{3}(\xi)+k_{2}\left(w^{\prime}(\xi)\right)^{2}}
$$

and

$$
\tilde{w}(\xi)=\frac{-B R+A w(\xi)}{A+B w(\xi)}
$$

where $A, B, a_{2}, b_{2}, c_{2}, d_{2}, f_{2}, k_{2}, m_{2}, l_{2}, r_{2}, a_{6}, b_{6}$ are arbitrary nonzero constants, and $w(\xi)$ is known solution of (2.4). The relationships among parameters are given by

$$
\begin{aligned}
& p_{2}=R\left(-b_{2}+m_{2}+f_{2} R\right), \\
& q_{2}=\frac{1}{k_{2} l_{2}}\left[b_{2} l_{2}^{2}-\left(l_{2}^{2}+k_{2}^{2} R\right)\left(m_{2}+r_{2}+\left(f_{2}+l_{2}\right) R\right)\right], \\
& n_{2}=\frac{1}{k_{2}}\left(f_{2} l_{2}-l_{2}^{2}-k_{2}^{2} R\right), \\
& d_{2}=-c_{2}+\frac{1}{k_{2}}\left(f_{2} l_{2}-l_{2}^{2}\right)+\frac{1}{l_{2}}\left(m_{2}+r_{2}+f_{2} R\right) k_{2}-k_{2} R, \\
& a_{2}=\frac{1}{k_{2}}\left[b_{2} l_{2}-l_{2}^{2} R-l_{2}\left(m_{2}+r_{2}+f_{2} R\right)-k_{2} R\left(c_{2}+k_{2} R\right)\right] .
\end{aligned}
$$


The combinations of an arbitrary solution to (2.4) with transformation (3.1) or (3.2) and iterations can result in infinite sequence solutions for (2.4). Here we only list three groups, and omit others.

$$
\begin{aligned}
& \left\{\begin{array}{l}
w_{n}(\xi)=\frac{p_{2}+q_{2} w_{n-1}(\xi)+m_{2} w_{n-1}^{2}(\xi)+r_{2} w_{n-1}^{\prime}(\xi)+n_{2} w_{n-1}^{3}(\xi)+l_{2}\left(w_{n-1}^{\prime}(\xi)\right)^{2}}{a_{2}+b_{2} w_{n-1}(\xi)+d_{2} w_{n-1}^{2}(\xi)+c_{2} w_{n-1}^{\prime}(\xi)+f_{2} w_{n-1}^{3}(\xi)+k_{2}\left(w_{n-1}^{\prime}(\xi)\right)^{2}} \\
w_{0}(\xi)=-\sqrt{-R} \tanh (\sqrt{-R} \xi), R<0, n=1,2, \cdots
\end{array}\right. \\
& \left\{\begin{array}{l}
w_{n}(\xi)=\frac{p_{2}+q_{2} w_{n-1}(\xi)+m_{2} w_{n-1}^{2}(\xi)+r_{2} w_{n-1}^{\prime}(\xi)+n_{2} w_{n-1}^{3}(\xi)+l_{2}\left(w_{n-1}^{\prime}(\xi)\right)^{2}}{a_{2}+b_{2} w_{n-1}(\xi)+d_{2} w_{n-1}^{2}(\xi)+c_{2} w_{n-1}^{\prime}(\xi)+f_{2} w_{n-1}^{3}(\xi)+k_{2}\left(w_{n-1}^{\prime}(\xi)\right)^{2}} \\
w_{0}(\xi)=\sqrt{R} \tan (\sqrt{R} \xi), R>0, n=1,2, \cdots
\end{array}\right.
\end{aligned}
$$

and

$$
\left\{\begin{array}{l}
w_{n}(\xi)=\frac{-B R+A w_{n-1}(\xi)}{A+B w_{n-1}(\xi)} \\
w_{1}(\xi)=\frac{\sqrt{R}\left[-2 a_{6} b_{6} \sqrt{R}+\left(a_{6}^{2}-b_{6}^{2} R\right)(\sec (2 \sqrt{R} \xi)+\tan (2 \sqrt{R} \xi))\right]}{a_{6}^{2}-b_{6}^{2} R+2 a_{6} b_{6} \sqrt{R}[\sec (2 \sqrt{R} \xi)+\tan (2 \sqrt{R} \xi)]} \\
R>0, n=2,3, \cdots
\end{array}\right.
$$

\subsection{Nonlinear superposition formulas}

Under the condition of $m_{3} d_{3}<0$, Eq. (2.4) possesses the following solutions $\tilde{w}(\xi)$,

$$
\begin{aligned}
& \tilde{w}(\xi)=\frac{\mathrm{i} R\left[\mathrm{i} m_{3} \sqrt{R}+\left(m_{3}+\mathrm{i} d_{3} \sqrt{R}+c_{3} R\right) w_{2}(\xi)+\left(-c_{3} R+d_{3} w_{2}(\xi)\right) w_{1}(\xi)\right]}{-\sqrt{R^{3}}\left(d_{3}+c_{3} w_{2}(\xi)\right)+\left(m_{3} \sqrt{R}+\mathrm{i} R d_{3}+c_{3} \sqrt{R^{3}}-\mathrm{i} m_{3} w_{2}(\xi)\right) w_{1}(\xi)}, \\
& \tilde{w}(\xi)=\frac{m_{3}+d_{3} w_{2}(\xi)+\frac{1}{\sqrt{R}}\left[-\mathrm{i} c_{3} R w_{1}(\xi)+\mathrm{i}\left(m_{3}+c_{3} R+d_{3} w_{1}(\xi)\right) w_{2}(\xi)\right]}{d_{3}+c_{3} w_{2}(\xi)-\frac{1}{\sqrt{R^{3}}}\left(m_{3} \sqrt{R}-\mathrm{i} R d_{3}+c_{3} \sqrt{R^{3}}+\mathrm{i} m_{3} w_{2}(\xi)\right) w_{1}(\xi)},
\end{aligned}
$$

and

$$
\tilde{w}(\xi)=\frac{R\left[-r_{3} w_{1}(\xi)+\left(p_{3}+r_{3}\right) w_{2}(\xi)-p_{3} w_{3}(\xi)\right]}{-r_{3} w_{2}(\xi) w_{3}(\xi)+w_{1}(\xi)\left(-p_{3} w_{2}(\xi)+\left(p_{3}+r_{3}\right) w_{3}(\xi)\right)}
$$

where $c_{3}, p_{3}, r_{3}$ are arbitrary nonzero constants, and $w_{1}(\xi), w_{2}(\xi), w_{3}(\xi)$ are three known solutions of (2.4). Thus, combining nonlinear superposition formulas (3.6) and (3.8) with known solutions, one gets

$$
\left\{\begin{array}{l}
w_{n}(\xi)=\frac{\mathrm{i} R\left[\mathrm{i} m_{3} \sqrt{R}+\left(m_{3}+\mathrm{i} d_{3} \sqrt{R}+c_{3} R\right) w_{n-1}(\xi)+\left(-c_{3} R+d_{3} w_{n-1}(\xi)\right) w_{n-2}(\xi)\right]}{-\sqrt{R^{3}}\left(d_{3}+c_{3} w_{n-1}(\xi)\right)+\left(m_{3} \sqrt{R}+\mathrm{i} R d_{3}+c_{3} \sqrt{R^{3}}-\mathrm{i} m_{3} w_{n-1}(\xi)\right) w_{n-2}(\xi)} \\
w_{1}(\xi)=-\sqrt{-R} \tanh (\sqrt{-R} \xi), \\
w_{2}(\xi)=\frac{b_{3} R+a_{3} \sqrt{-R} \tanh (\sqrt{-R} \xi)}{-a_{3}+b_{3} \sqrt{-R} \tanh (\sqrt{-R} \xi)}, \quad n=3,4, \cdots
\end{array}\right.
$$


and

$$
\left\{\begin{aligned}
w_{n}(\xi) & =\frac{R\left[-r_{3} w_{n-3}(\xi)+\left(p_{3}+r_{3}\right) w_{n-2}(\xi)-p_{3} w_{n-1}(\xi)\right]}{-r_{3} w_{n-2}(\xi) w_{n-1}(\xi)+w_{n-3}(\xi)\left(-p_{3} w_{n-2}(\xi)+\left(p_{3}+r_{3}\right) w_{n-1}(\xi)\right)} \\
w_{1}(\xi) & =\frac{-b_{5} R+a_{5} \sqrt{R}[\sec (2 \sqrt{R} \xi)+\tan (2 \sqrt{R} \xi)]}{a_{5}+b_{5} \sqrt{R}[\sec (2 \sqrt{R} \xi)+\tan (2 \sqrt{R} \xi)]} \\
w_{2}(\xi) & =\frac{\sqrt{R}[\cos (\sqrt{R} \xi)+\sin (\sqrt{R} \xi)]}{\cos (\sqrt{R} \xi)-\sin (\sqrt{R} \xi)} \\
w_{3}(\xi) & =\sqrt{R} \tan (\sqrt{R} \xi), \quad n=4,5, \ldots
\end{aligned}\right.
$$

\section{Application to space-time FSRLW equation}

Now, we focus on the space-time FSRLW equation [8]

$$
D_{t}^{2 \alpha} u+D_{x}^{2 \alpha} u+u D_{t}^{\alpha}\left(D_{x}^{\alpha} u\right)+D_{x}^{\alpha} u D_{t}^{\alpha} u+D_{t}^{2 \alpha}\left(D_{x}^{2 \alpha} u\right)=0, \quad 0<\alpha \leq 1,
$$

which arises in several physical applications including ion sound waves in plasma. When $\alpha=1$, it is shown that this equation describes weakly nonlinear ion acoustic and spacecharge waves, and the real-valued $u(x, t)$ corresponds to the dimensionless fluid velocity with a decay condition.

Applying the transformation

$$
u(x, t)=U(\xi)=U\left(\frac{k x^{\alpha}}{\Gamma(1+\alpha)}+\frac{c t^{\alpha}}{\Gamma(1+\alpha)}\right)
$$

to Eq. (4.1), integrating twice with respect to $\xi$, and taking the integration constants as zero, we arrive at

$$
\left(c^{2}+k^{2}\right) U+\frac{c k}{2} U^{2}+c^{2} k^{2} U^{\prime \prime}=0 .
$$

Analyzing $U^{\prime \prime}$ and $U^{2}$ in (4.2) reveals $m=2$. Therefore, we assume that

$$
\begin{aligned}
U(\xi)=A_{0}+ & A_{1} w(\xi)+C_{1} \sqrt{R+w^{2}(\xi)}+\frac{B_{1}}{w(\xi)}+A_{2} w^{2}(\xi) \\
& +C_{2} w(\xi) \sqrt{R+w^{2}(\xi)}+\frac{B_{2}}{w^{2}(\xi)}
\end{aligned}
$$

where $A_{0}, A_{1}, A_{2}, B_{1}, B_{2}, C_{1}, C_{2}$, and $R$ are undetermined constants.

The substitution of expression (4.3) along with (2.4) into (4.2), then multiplication by $w^{4}(\xi) \sqrt{R+w^{2}(\xi)}$ and collection of the same powers of $w^{j_{1}}(\xi)$ and $w^{j_{2}}(\xi)\left(\sqrt{R+w^{2}(\xi)}\right)^{j_{3}}$ lead to a sequence of nonlinear algebraic equations whose solutions can be computed 
with the aid of Maple as

Case 1:

$$
\left\{\begin{array}{l}
R=\frac{k^{2}+c^{2}}{c^{2} k^{2}}, A_{0}=-\frac{6\left(k^{2}+c^{2}\right)}{c k}, A_{1}=0, A_{2}=-6 c k, \\
B_{1}=0, B_{2}=0, C_{1}=0, C_{2}= \pm 6 c k .
\end{array}\right.
$$

Case 2:

$$
\left\{\begin{array}{l}
R=-\frac{k^{2}+c^{2}}{c^{2} k^{2}}, A_{0}=\frac{4\left(k^{2}+c^{2}\right)}{c k}, A_{1}=0, A_{2}=-6 c k, \\
B_{1}=0, B_{2}=0, C_{1}=0, C_{2}= \pm 6 c k .
\end{array}\right.
$$

Case 3:

$$
\left\{\begin{array}{l}
R=\frac{k^{2}+c^{2}}{4 c^{2} k^{2}}, A_{0}=-\frac{3\left(k^{2}+c^{2}\right)}{c k}, A_{1}=0, A_{2}=-12 c k, \\
B_{1}=0, B_{2}=0, C_{1}=0, C_{2}=0 .
\end{array}\right.
$$

Case 4:

$$
\left\{\begin{array}{l}
R=-\frac{k^{2}+c^{2}}{4 c^{2} k^{2}}, A_{0}=\frac{k^{2}+c^{2}}{c k}, A_{1}=0, A_{2}=-12 c k, \\
B_{1}=0, B_{2}=0, C_{1}=0, C_{2}=0 .
\end{array}\right.
$$

Case 5:

Case 6:

$$
\left\{\begin{array}{l}
R=-\frac{k^{2}+c^{2}}{16 c^{2} k^{2}}, A_{0}=-\frac{k^{2}+c^{2}}{2 c k}, A_{1}=0, A_{2}=-12 c k, \\
B_{1}=0, B_{2}=-\frac{3\left(c^{4}+2 c^{2} k^{2}+k^{4}\right)}{64 c^{3} k^{3}}, C_{1}=0, C_{2}=0 .
\end{array}\right.
$$

Case 7:

$$
\left\{\begin{array}{l}
R=\frac{k^{2}+c^{2}}{16 c^{2} k^{2}}, A_{0}=-\frac{3\left(k^{2}+c^{2}\right)}{2 c k}, A_{1}=0, A_{2}=-12 c k, \\
B_{1}=0, B_{2}=-\frac{3\left(c^{4}+2 c^{2} k^{2}+k^{4}\right)}{64 c^{3} k^{3}}, C_{1}=0, C_{2}=0 .
\end{array}\right.
$$

Case 8:

$$
\left\{\begin{array}{l}
R=\frac{k^{2}+c^{2}}{4 c^{2} k^{2}}, A_{0}=-\frac{3\left(k^{2}+c^{2}\right)}{c k}, A_{1}=0, A_{2}=0 \\
B_{1}=0, B_{2}=-\frac{3\left(k^{2}+c^{2}\right)^{2}}{4 c^{3} k^{3}}, C_{1}=0, C_{2}=0 .
\end{array}\right.
$$

$$
\left\{\begin{array}{l}
R=-\frac{k^{2}+c^{2}}{4 c^{2} k^{2}}, A_{0}=\frac{k^{2}+c^{2}}{c k}, A_{1}=0, A_{2}=0 \\
B_{1}=0, B_{2}=-\frac{3\left(k^{2}+c^{2}\right)^{2}}{4 c^{3} k^{3}}, C_{1}=0, C_{2}=0 .
\end{array}\right.
$$

In fact, we note $R=\frac{k^{2}+c^{2}}{c^{2} k^{2}}>0$ from Case 1 , which means that we can get trigonometric function solutions to Eq. (4.1). The expression (4.3) can be rewritten as

$$
U_{1}(\xi)=-\frac{6\left(k^{2}+c^{2}\right)}{c k}-6 c k w^{2}(\xi) \pm 6 c k w(\xi) \sqrt{R+w^{2}(\xi)} .
$$


Thus, by substituting (2.8)-(2.14) into (4.4) respectively, a series of general exact solutions can be obtained. Here we only list one of them via inserting (2.8) into (4.4), namely,

$$
\begin{array}{r}
U_{11}(\xi)=-\frac{6\left(k^{2}+c^{2}\right)}{c k}-\frac{6\left(k^{2}+c^{2}\right)}{c k} \tan ^{2}\left(\sqrt{\frac{k^{2}+c^{2}}{c^{2} k^{2}}} \xi\right) \\
\pm 6 c k \sqrt{\frac{k^{2}+c^{2}}{c^{2} k^{2}}} \tan \left(\sqrt{\frac{k^{2}+c^{2}}{c^{2} k^{2}}} \xi\right) \sqrt{\frac{k^{2}+c^{2}}{c^{2} k^{2}} \sec ^{2}\left(\sqrt{\frac{k^{2}+c^{2}}{c^{2} k^{2}}} \xi\right)}
\end{array}
$$

where $\xi=\frac{k x^{\alpha}}{\Gamma(1+\alpha)}+\frac{c t^{\alpha}}{\Gamma(1+\alpha)}$.

Moreover, it is clear that many trigonometric function solutions corresponding to Cases 3, 6, and 7 can be given in a similar way. But for brevity we do not list all of them.

According to Case 2, we can get the following expression

$$
U_{2}(\xi)=\frac{4\left(k^{2}+c^{2}\right)}{c k}-6 c k w^{2}(\xi) \pm 6 c k w(\xi) \sqrt{R+w^{2}(\xi)}
$$

In consideration of $R=-\frac{k^{2}+c^{2}}{c^{2} k^{2}}<0$, some hyperbolic function solutions can be derived by carrying (2.5)-(2.7) into (4.5). The substitution of (2.5) into (4.5) yields

$$
\begin{array}{r}
U_{21}(\xi)=\frac{4\left(k^{2}+c^{2}\right)}{c k}-\frac{6\left(k^{2}+c^{2}\right)}{c k} \tanh ^{2}\left(\sqrt{\frac{k^{2}+c^{2}}{c^{2} k^{2}}} \xi\right) \mp 6 c k \sqrt{\frac{k^{2}+c^{2}}{c^{2} k^{2}}} \\
\quad \times \tanh \left(\sqrt{\frac{k^{2}+c^{2}}{c^{2} k^{2}}} \xi\right) \sqrt{-\frac{k^{2}+c^{2}}{c^{2} k^{2}}+\frac{k^{2}+c^{2}}{c^{2} k^{2}} \tanh ^{2}\left(\sqrt{\frac{k^{2}+c^{2}}{c^{2} k^{2}}} \xi\right)}
\end{array}
$$

where $\xi=\frac{k x^{\alpha}}{\Gamma(1+\alpha)}+\frac{c t^{\alpha}}{\Gamma(1+\alpha)}$.

Similarly, we can express many hyperbolic function solutions from Cases 4, 5, and 8 , whose details we omit here.

Next, we would like to present infinite sequence solutions to (4.1) based on Bäcklund transformations (3.1)-(3.2) and nonlinear superposition formulas (3.6) and (3.8). Combining (3.4) with (4.4), we have

$$
\left\{\begin{array}{l}
u_{n}(x, t)=U_{n}(\xi)=-\frac{6\left(k^{2}+c^{2}\right)}{c k}-6 c k w_{n}^{2}(\xi) \pm 6 c k w_{n}(\xi) \sqrt{R+w_{n}^{2}(\xi)} \\
w_{n}(\xi)=\frac{p_{2}+q_{2} w_{n-1}(\xi)+m_{2} w_{n-1}^{2}(\xi)+r_{2} w_{n-1}^{\prime}(\xi)+n_{2} w_{n-1}^{3}(\xi)+l_{2}\left(w_{n-1}^{\prime}(\xi)\right)^{2}}{a_{2}+b_{2} w_{n-1}(\xi)+d_{2} w_{n-1}^{2}(\xi)+c_{2} w_{n-1}^{\prime}(\xi)+f_{2} w_{n-1}^{3}(\xi)+k_{2}\left(w_{n-1}^{\prime}(\xi)\right)^{2}} \\
w_{0}(\xi)=\sqrt{R} \tan (\sqrt{R} \xi)
\end{array}\right.
$$

where $\xi=\frac{k x^{\alpha}}{\Gamma(1+\alpha)}+\frac{c t^{\alpha}}{\Gamma(1+\alpha)}, R=\frac{k^{2}+c^{2}}{c^{2} k^{2}}>0, n=1,2, \ldots$ 
Combining (3.5) with (4.4), we have

$$
\left\{\begin{array}{l}
u_{n}(x, t)=U_{n}(\xi)=-\frac{6\left(k^{2}+c^{2}\right)}{c k}-6 c k w_{n}^{2}(\xi) \pm 6 c k w_{n}(\xi) \sqrt{R+w_{n}^{2}(\xi)} \\
w_{n}(\xi)=\frac{-B R+A w_{n-1}(\xi)}{A+B w_{n-1}(\xi)} \\
w_{1}(\xi)=\frac{\sqrt{R}\left[-2 a_{6} b_{6} \sqrt{R}+\left(a_{6}^{2}-b_{6}^{2} R\right)(\sec (2 \sqrt{R} \xi)+\tan (2 \sqrt{R} \xi))\right]}{a_{6}^{2}-b_{6}^{2} R+2 a_{6} b_{6} \sqrt{R}[\sec (2 \sqrt{R} \xi)+\tan (2 \sqrt{R} \xi)]}
\end{array}\right.
$$

where $\xi=\frac{k x^{\alpha}}{\Gamma(1+\alpha)}+\frac{c t^{\alpha}}{\Gamma(1+\alpha)}, R=\frac{k^{2}+c^{2}}{c^{2} k^{2}}>0, n=2,3, \cdots$

Combining (3.3) with (4.5), we have

$$
\left\{\begin{array}{l}
u_{n}(x, t)=U_{n}(\xi)=\frac{4\left(k^{2}+c^{2}\right)}{c k}-6 c k w_{n}^{2}(\xi) \pm 6 c k w_{n}(\xi) \sqrt{R+w_{n}^{2}(\xi)}, \\
w_{n}(\xi)=\frac{p_{2}+q_{2} w_{n-1}(\xi)+m_{2} w_{n-1}^{2}(\xi)+r_{2} w_{n-1}^{\prime}(\xi)+n_{2} w_{n-1}^{3}(\xi)+l_{2}\left(w_{n-1}^{\prime}(\xi)\right)^{2}}{a_{2}+b_{2} w_{n-1}(\xi)+d_{2} w_{n-1}^{2}(\xi)+c_{2} w_{n-1}^{\prime}(\xi)+f_{2} w_{n-1}^{3}(\xi)+k_{2}\left(w_{n-1}^{\prime}(\xi)\right)^{2}} \\
w_{0}(\xi)=-\sqrt{-R} \tanh (\sqrt{-R} \xi)
\end{array}\right.
$$

where $\xi=\frac{k x^{\alpha}}{\Gamma(1+\alpha)}+\frac{c t^{\alpha}}{\Gamma(1+\alpha)}, R=-\frac{k^{2}+c^{2}}{c^{2} k^{2}}<0, n=1,2, \cdots$

Combining (3.10) with (4.4), we have

$$
\left\{\begin{array}{l}
u_{n}(x, t)=U_{n}(\xi)=-\frac{6\left(k^{2}+c^{2}\right)}{c k}-6 c k w_{n}^{2}(\xi) \pm 6 c k w_{n}(\xi) \sqrt{R+w_{n}^{2}(\xi)} \\
w_{n}(\xi)=\frac{R\left[-r_{3} w_{n-3}(\xi)+\left(p_{3}+r_{3}\right) w_{n-2}(\xi)-p_{3} w_{n-1}(\xi)\right]}{-r_{3} w_{n-2}(\xi) w_{n-1}(\xi)+w_{n-3}(\xi)\left(-p_{3} w_{n-2}(\xi)+\left(p_{3}+r_{3}\right) w_{n-1}(\xi)\right)} \\
w_{1}(\xi)=\frac{-b_{5} R+a_{5} \sqrt{R}[\sec (2 \sqrt{R} \xi)+\tan (2 \sqrt{R} \xi)]}{a_{5}+b_{5} \sqrt{R}[\sec (2 \sqrt{R} \xi)+\tan (2 \sqrt{R} \xi)]} \\
w_{2}(\xi)=\frac{\sqrt{R}[\cos (\sqrt{R} \xi)+\sin (\sqrt{R} \xi)]}{\cos (\sqrt{R} \xi)-\sin (\sqrt{R} \xi)} \\
w_{3}(\xi)=\sqrt{R} \tan (\sqrt{R} \xi)
\end{array}\right.
$$

where $\xi=\frac{k x^{\alpha}}{\Gamma(1+\alpha)}+\frac{c t^{\alpha}}{\Gamma(1+\alpha)}, R=\frac{k^{2}+c^{2}}{c^{2} k^{2}}>0, n=4,5, \cdots$

\section{Conclusion}

To sum up, taking advantage of Bäcklund transformations and nonlinear superposition formulas of solutions to Riccati equation, we have successfully established infinite sequence solutions for space-time fractional symmetric regularized long wave equation through symbolic computation. This method can be extended to deal with other nonlinear fractional partial differential equations. 


\section{Acknowledgments}

This work is supported by the National Natural Science Foundation of China (Grant No.11462019) and the Scientific Research Foundation of Inner Mongolia University for Nationalities (Grant No. NMD1306). The author would like to thank the referees for their time and comments.

\section{References}

[1] Wazwaz A. M. and Ebaid A., A study on couplings of the fifth-order integrable SawadaKotera and Lax equations. Romanian Journal of Physics, 59 (5-6) (2014), 454-465.

[2] Wazwaz A. M., New (3+1)-dimensional nonlinear evolution equations with Burgers and Sharma-Tasso-Olver equations constituting the main parts. Proceedings of the Romanian Academy, Series A, 16 (2015), 32-40.

[3] Zhang S., Zong Q. A., Liu D. and Gao Q., A generalized exp-function method for fractional Riccati differential equations. Communications in Fractional Calculus, 1 (2010), 48-51.

[4] Triki H., Mirzazadeh M., Bhrawy A. H., Razborova P. and Biswas A., Solitons and other solutions to long-wave short-wave interaction equation. Romanian Journal of Physics, 60 (1-2) (2015), 72-86.

[5] Zhang Z. Y., Zhong J., Dou S. S., Liu J., Peng D. and Gao T., First integral method and exact solutions to nonlinear partial differential equations arising in mathematical physics. Romanian Reports in Physics, 65 (4) (2013), 1155-1169.

[6] Jafari H., Tajadodi H. and Baleanu D., Application of a homogeneous balance method to exact solutions of nonlinear fractional evolution equations. Journal of Computational and Nonlinear Dynamics, 9 (2014), 021019, 4 pages.

[7] Jafari H., Tajadodi H., Baleanu D., Al-Zahrani A. A., Alhamed Y. A. and Zahid A. H., Exact solutions of Boussinesq and KdV-mKdV equations by fractional sub-equation method. Romanian Reports in Physics, 65 (4) (2013), 1119-1124.

[8] Alzaidy J. F., The fractional sub-equation method and exact analytical solutions for some nonlinear fractional PDEs. American Journal of Mathematical Analysis, 1 (2013), 14-19.

[9] Wang G. W. and Xu T. Z., The modified fractional sub-equation method and its applications to nonlinear fractional partial differential equations. Romanian Journal of Physics, 59 (7-8) (2014), 636-645.

[10] Wang G. W. and Xu T. Z., The improved fractional sub-equation method and its applications to nonlinear fractional partial differential equations. Romanian Reports in Physics, 66 (3) (2014), 595-602.

[11] Bekir A., Aksoy E. and Cevikel A. C., Exact solutions of nonlinear time fractional partial differential equations by sub-equation method. Mathematical Methods in the Applied Sciences, 38 (13) (2015), 2779-2784.

[12] Güner Ö., Bekir A. and Bilgil H., A note on exp-function method combined with complex transform method applied to fractional differential equations. Advances in Nonlinear Analysis, 4 (3) (2015), 201-208.

[13] Zheng B., $\left(G^{\prime} / G\right)$-expansion method for solving fractional partial differential equations in the theory of mathematical physics. Communications in Theoretical Physics, 58 (5) (2012), 623630 . 
[14] Bekir A. and Güner Ö., Exact solutions of nonlinear fractional differential equations by $\left(G^{\prime} / G\right)$-expansion method. Chinese Physics B, 22 (11) (2013), 110202, 6 pages.

[15] Bekir A., Güner Ö. and Ünsal Ö., The first integral method for exact solutions of nonlinear fractional differential equations. Journal of Computational and Nonlinear Dynamics, 10 (2015), 021020, 5 pages.

[16] Bulut H., Baskonus H. M. and Pandir Y., The modified trial equation method for fractional wave equation and time fractional generalized Burgers equation. Abstract and Applied Analysis, (2013), Article ID 636802, 8 pages.

[17] Zheng B. and Feng Q. H., The Jacobi elliptic equation method for solving fractional partial differential equations. Abstract and Applied Analysis, (2014), Article ID 249071, 9 pages.

[18] Ege S. M. and Misirli E., The modified Kudryashov method for solving some fractional-order nonlinear equations. Advances in Difference Equations, 135 (2014), 13 pages.

[19] Kumar D., Singh J. and Sushila, Application of homotopy analysis transform method to fractional biological population model. Romanian Reports in Physics, 65 (1) (2013), 63-75.

[20] Wu G. C. and Lee E. W. M., Fractional variational iteration method and its application. Physics Letters A, 374 (25) (2010), 2506-2509.

[21] El-Sayed A. M. A. and Gaber M., The Adomian decomposition method for solving partial differential equations of fractal order in finite domains. Physics Letters A, 359 (3) (2006), 175182.

[22] Li Z. B. and He J. H., Fractional complex transform for fractional differential equations. Mathematical and Computational Applications, 15 (5) (2010), 970-973.

[23] Jumarie G., Modified Riemann-Liouville derivative and fractional Taylor series of nondifferentiable functions further results. Computers and Mathematics with Applications, 51 (2006), 1367-1376.

[24] Lu B., Bäcklund transformation of fractional Riccati equation and infinite sequence solutions of nonlinear fractional PDEs. Abstract and Applied Analysis, (2014), Article ID 572052, 6 pages.

[25] Taogetusang, Infinite sequence peak solitary wave solutions of $\mathrm{CH}-\mathrm{r}$ equation by an auxiliary equation. Chinese Journal of Engineering Mathematics, 29 (6) (2012), 865-876. 